\title{
Electromagnetic design for SuperSpec; a lithographically-patterned millimetre-wave spectrograph
}

\author{
P. S. Barry ${ }^{a}$, E. Shirokoff ${ }^{b}$, A. Kovács ${ }^{c}$, T. J. Reck ${ }^{d}$, S. Hailey-Dunsheath ${ }^{b}$, C. M. McKenney ${ }^{b}$, \\ L. J. Swenson ${ }^{b, c}$, M. I. Hollister ${ }^{b, c}$ H. G. Leduc ${ }^{c}$, S. Doyle ${ }^{a}$, R. O'Brient ${ }^{b}$, N. Llombart ${ }^{f}$, D.

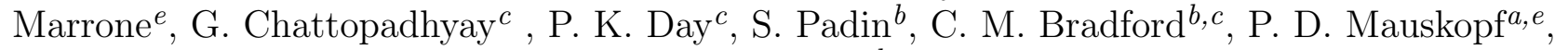 \\ J. Zmuidzinas ${ }^{b, c}$ \\ ${ }^{a}$ Department of Physics and Astronomy, Cardiff University, Cardiff CF24 3YB, UK \\ ${ }^{b}$ California Institute of Technology, 320-47, Pasadena CA 91125, USA \\ ${ }^{c}$ University of Minnesota, 116 Church St SE, Minneapolis, MN 55414, USA \\ $d$ Jet Propulsion Laboratory, 320-231, Pasadena, CA 91125, USA \\ ${ }^{e}$ University of Arizona, 933 North Cherry Avenue, Tucson, AZ 85721, USA \\ f Optics Department, Universidad Complutense de Madrid, Madrid, Spain \\ ${ }^{e}$ Arizona State University, 650 E. Tyler Mall, Tempe, AZ 85287, USA
}

\begin{abstract}
SuperSpec is an innovative, fully planar, compact spectrograph for $\mathrm{mm} / \mathrm{sub}-\mathrm{mm}$ astronomy. SuperSpec is based on a superconducting filter-bank consisting of a series of planar half-wavelength filters to divide up the incoming, broadband radiation. The power in each filter is then coupled into titanium nitride lumped element kinetic inductance detectors, facilitating the read out of a large number of filter elements. We will present electromagnetic simulations of the different components that will make up an $\mathcal{R}=700$ prototype instrument. Based on these simulations, we discuss optimisation of the coupling between the antenna, transmission line, filters and detectors.
\end{abstract}

Keywords: SuperSpec, On-chip Spectrometer, Kinetic Inductance Detector, Filter-bank

\section{INTRODUCTION}

Submillimetre galaxies are a population of massive star-forming galaxies containing luminous dust emission at FIR-submm wavelengths observable out to high redshifts $(z>1)$. Current Herschel surveys (H-ATLAS, ${ }^{1}$ HerMES $^{2}$ ) are identifying large numbers of these bright submillimetre objects, and accurate identification of the redshifts of these galaxies is essential to improving our understanding of star formation and galaxy evolution. Many of these sources cannot be identified with an optical counterpart with known redshift. Submillimetre photometric measurements provide a fast method of estimating redshift, but this approach is inaccurate due to the uncertainty of the dust temperature. Spectroscopic measurements of the numerous lines that lie within the submillimetre band is the preferred method of determining redshifts. Currently, measurement of these submillimetre spectral lines is carried out by grating spectrometers (Z-Spec, ${ }^{3}$ Zeus, ${ }^{4}$ Zeus- $\mathrm{II}^{5}$ ), which are bulky instruments that are limited in instantaneous bandwidth and mapping speed. The most efficient method of obtaining these redshifts would be to have a multi-object spectrometer consisting of hundreds/thousands of pixels, each measuring a spectrum.

Advances in submillimetre astronomical instrumentation over the past twenty years have been substantial; progressing from instruments with tens of pixels in the early 90s to large format arrays consisting of tens of thousands of pixels. Assuming that the current growth rate continues, arrays of $10^{5}$ pixels are forecast to be available by the end of the decade. The most promising detector technology that has the potential to realise these large format cameras is the superconducting kinetic inductance detector ${ }^{6}$ (KID). In this device, photon absorption breaks Cooper pairs generating quasiparticles. This results in a perturbation of the complex conductivity $\delta \sigma(\omega)=\delta \sigma_{1}-j \delta \sigma_{2}$ producing a measurable shift in the resonator dissipation and frequency.

Further author information:

E-mail: peter.barry@astro.cf.ac.uk; Telephone: (+44) 29-2087-0609

Millimeter, Submillimeter, and Far-Infrared Detectors and Instrumentation for Astronomy VI, edited by Wayne S. Holland, Jonas Zmuidzinas, Proc. of SPIE Vol. 8452

84522F @ 2012 SPIE - CCC code: 0277-786X/12/\$18 - doi: 10.1117/12.927089

Proc. of SPIE Vol. $845284522 \mathrm{~F}-1$ 
This paper outlines some of the design aspects of a novel on-chip spectrometer, which will be capable of measuring submillimetre spectra with a spectral resolution of $\mathcal{R} \sim 1000$, with an instrument that is more than 100 times more compact than current submillimetre spectrographs.

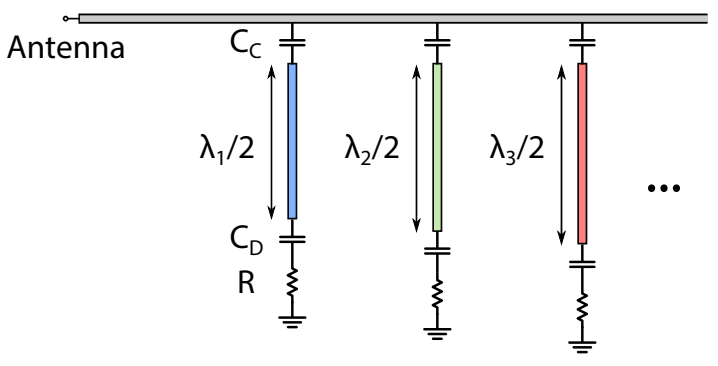

Figure 1. Simplified schematic of a filter bank spectrometer.

A schematic of such an on-chip spectrometer is shown in Figure 1. A broadband antenna couples incident radiation onto a superconducting planar transmission line. Placed along the feedline are a number of halfwavelength resonant filters that form the filter bank, with each filter extracting a narrow spectral band $\Delta \nu$ centred on the resonant frequency $\nu_{0}$. The power contained in each filter is then coupled into the absorbing element of a lumped element kinetic inductance detector (LEKID). ${ }^{7}$ Over the past decade KID technology has matured with numerous major projects approaching background-limited performance (NIKA, ${ }^{8}$ MUSIC $^{9}$ ). The intense research interest in KIDs has been primarily driven by their intrinsic compatibility with frequency domain multiplexing. Multiplexing factors of 256 have been demonstrated ${ }^{10}$ and more than a thousand pixel readout per wire is expected to be achievable in the near future. ${ }^{11,12}$

The choice of materials for each component of the spectrometer is constrained by a number of practical considerations. The main feedline must be made from material with a superconducting energy gap above photon energy at the highest optical frequency of interest, otherwise loss due to quasiparticle dissipation becomes prohibitive. The choice of material therefore sets an upper limit on the frequency of operation of the spectrometer which corresponds to the gap frequency of the material. For submillimetre astronomy this restricts the choice of material to ones with $T_{c}>4.5 \mathrm{~K}$. Niobium fulfils this requirement with a gap frequency of $\nu_{g} \sim 700 \mathrm{GHz}$ and has been chosen as a baseline for the initial test device. The use of nitride materials such as NbN, NbTiN would enable the frequency of operation to be increased above $1 \mathrm{THz}$ and will be investigated in future design iterations. In contrast with the main feedline and filter resonators, the readout resonator must be dissipative at the signal frequencies of interest (hundreds of $\mathrm{GHz}$ ) and superconducting at the readout frequencies (hundreds of $\mathrm{MHz}$ ) in order to achieve efficient detection. This suggests an optimal transition temperature of approximately $1<T_{c}<4 \mathrm{~K}$.

The required number of filters to fully sample the bandwidth is given by

$$
N_{c}=\Sigma \mathcal{R} \ln \left(f_{u} / f_{l}\right),
$$

where $f_{u}$ and $f_{l}$ are the upper and lower frequencies of the band of interest, $\Sigma \geq 1$ is the spectral oversampling factor and determines the efficiency of the spectrometer. A higher $\Sigma$ value will result in increased power absorption for a given frequency, but will require more detector channels to read out the increased number of filters. For a prototype, Nyquist-sampled $(\Sigma=2), \mathcal{R}=700$ device, the required number of detectors is $N_{c} \sim 1400$. Based on the expected multiplexing density achievable with KIDs, this suggests the possibility that an entire spectrometer chip can be read out using a single measurement line.

\section{FILTER-BANK DESIGN}

Initial designs employ a thin-film microstrip architecture for the filter-bank, which has several advantages over alternative layouts such as CPW. Primarily, the proximity of the ground plane provides a natural means of stray light control, shielding the LEKIDs from direct illumination. This is vital for spectrometer operation as 
any parasitic broadband power absorbed by the LEKID must remain negligible compared to the signal power admitted by the resonant filter. The main disadvantage is that the design requires the deposition of a thin-film dielectric that has stringent requirements on the loss tangent to achieve the desired performance. For the initial design, we have used a $0.5 \mu \mathrm{m}$ SiN layer as the dielectric which has been successfully demonstrated in test devices by several mm-wavelength instrument groups at JPL and Caltech.

A schematic of a single filter is shown in the inset of Figure 2. The half wavelength filter is bent into the shape of a ' $U$ ' primarily to facilitate the coupling of power into the LEKID, but has also been shown to reduce the radiation loss from the resonator. ${ }^{13}$

The spectrometer resolution $\mathcal{R}$ is determined by the quality factor of the filter elements and is given by

$$
\frac{1}{Q_{\text {filt }}}=\frac{1}{Q_{\text {feed }}}+\frac{1}{Q_{i}}+\frac{1}{Q_{\text {det }}}=\mathcal{R}^{-1}
$$

where $Q_{\text {feed }}$ is the coupling quality factor which describes the strength of the coupling to external circuitry, $Q_{i}$ is the internal quality factor which accounts for the dissipative losses such as the conductor, dielectric or radiation losses, and $Q_{\text {det }}$ is the quality factor associated with the absorption of mm-wave power in the detector (cf. Section 3). In practice $Q_{i}>>Q_{\text {det }}$ and Equation 2 reduces to

$$
\frac{1}{Q_{\text {filt }}}=\frac{1}{Q_{\text {feed }}}+\frac{1}{Q_{\mathrm{det}}} .
$$

An analytical expression for the coupling quality factor for the ' $U$ ' resonator can be derived by modelling the coupling length $l_{c}$ (cf. inset of Figure 2) as a pair of proximately coupled microstrip lines ${ }^{14}$ and calculating the power leakage from the resonator into the feedline. The result is given by, ${ }^{15}$

$$
Q_{\text {feed }}=\frac{4 l_{r e s}^{2}}{\pi l_{c}^{2}} \frac{1}{I\left(\theta_{c}\right)},
$$

where

$$
\begin{gathered}
I\left(\theta_{c}\right)=\left(\frac{L_{\Delta}}{L_{\Sigma}}\right)^{2}\left(1+\operatorname{sinc} 2 \theta_{c}\right)^{2}+\left(\frac{C_{\Delta}}{C_{\Sigma}}\right)^{2}\left(1-\operatorname{sinc} 2 \theta_{c}\right)^{2}+2 \frac{L_{\Delta}}{L_{\Sigma}} \frac{C_{\Delta}}{C_{\Sigma}}\left(1-\operatorname{sinc}^{2} 2 \theta_{c}\right) \\
\theta_{c}=\frac{\pi l_{c}}{2 l_{\text {res }}}
\end{gathered}
$$

where $C_{\Sigma}$ and $C_{\Delta}$ are, respectively, the sum and difference of the even and odd mode per unit length capacitances, and $L_{\Sigma}$ and $L_{\Delta}$ are the corresponding per unit length inductances. Equation 4 was compared to a set of Sonnet simulations and is plotted in Figure 2. The discrepancy between the simulation and Equation 4 at small gap sizes may be due to the relative decrease in simulation accuracy due to the cell size being kept constant for all the simulations.

A simulation of a five-element filter-bank is shown in Figure 3, where the difference in length of adjacent resonators is constant and given by $\Delta l_{\text {res }}$. The effect of two different values of $\Delta l_{\text {res }}(=0.2,0.4 \mu \mathrm{m})$ demonstrates how spacing the filters closer together in frequency decreases the frequency ripple of the pass band, increasing the overall efficiency of the filter-bank.

The length difference between resonators is approaching the limit of the constraints set by photo-lithography. The ultimate performance of a real device will be determined by the dimensional uncertainty limited by the fabrication capabilities. The Deep UV lithographic stepper at JPL can reproducibly achieve $0.1 \mu \mathrm{m}$ tolerances for features with a minimum reliable line width and gap size of $1 \mu \mathrm{m}$. To determine how fabrication tolerances would affect the spectrometer performance, a set of simulations were carried out varying a single dimension over a typical range of values, while keeping all other dimensions at their nominal values. The simulation layout used for the tolerance analysis is shown in Figure 4. Table 1 summarises the results of the tolerance analysis for a range of values that are considered to be achievable using the JPL fabrication facilities. The analysis included both the ' $U$ ' resonator and $\mathrm{mm}$-wave absorber to accurately model the contributions from all of the main mm-wave components. 


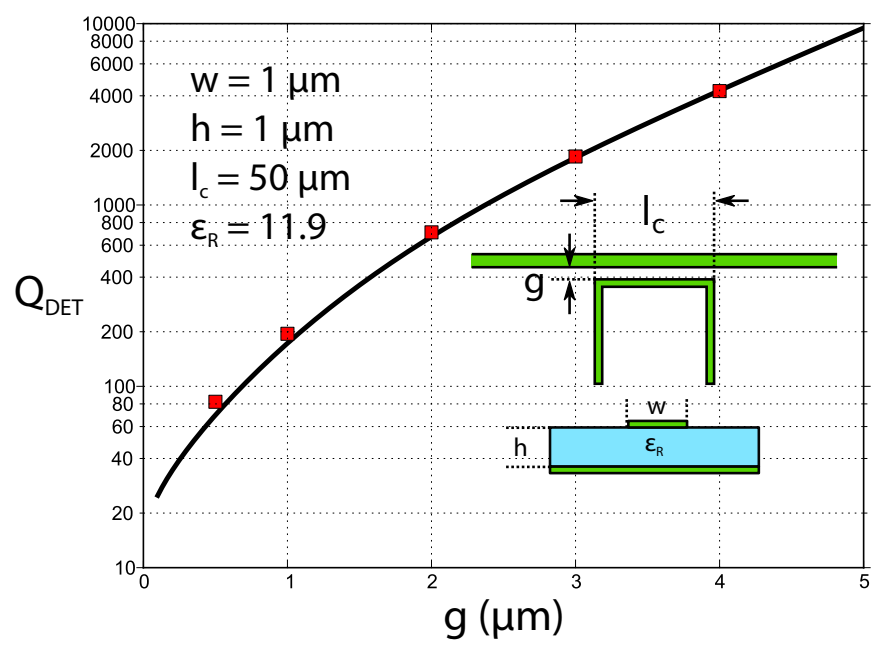

Figure 2. Plot of simulated (red squares) and theoretical (black line) values of $Q_{\text {feed }}$ for a range of gap sizes for a resonator length $l_{\text {res }}=200 \mu \mathrm{m}$.

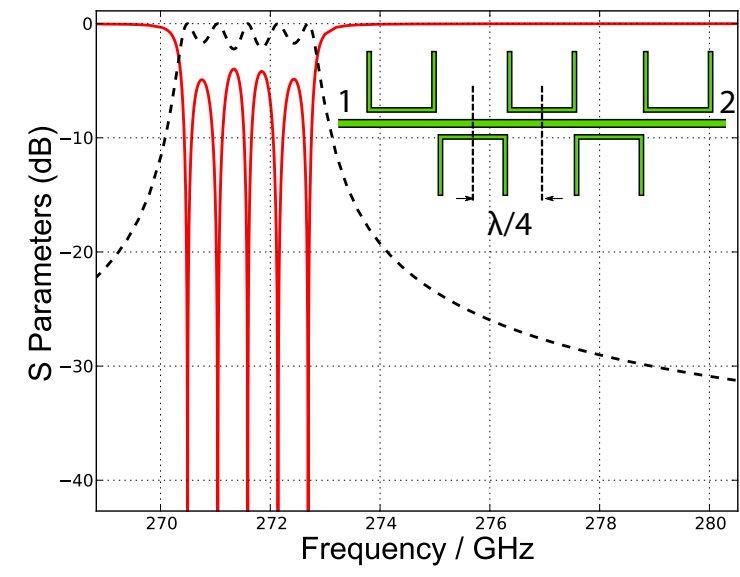

(a) $\Delta l_{\text {res }}=0.4 \mu \mathrm{m}, \Sigma=1.9$. (inset) Schematic layout of the simulation

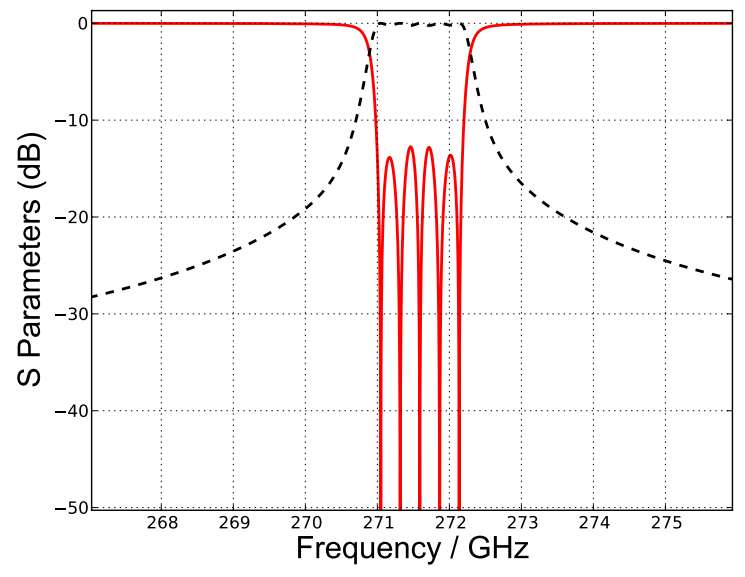

(b) $\Delta l_{\text {res }}=0.2 \mu \mathrm{m}, \Sigma=3.1$

Figure 3. Simulation of the scattering parameters $\left(S_{21}\right.$ - red solid line, $S_{11}$-black dashed line) of a signal past a five-element filter-bank to demonstrate the effect of increasing $\Sigma$.

The main dimension that requires strict control is the feedline to filter coupling gap $(g)$ which determines the coupling quality factor and ultimately the spectrometer resolution. A $\pm 0.1 \mu \mathrm{m}$ variation in gap translates to a $20 \%$ variation in $Q_{\text {feed }}$, so tight control over the etching process during fabrication is essential. Variations in the width of the feedline and resonator line have a similar effect on $Q_{\text {feed }}$.

The dielectric properties of the SiN layer also require fine control. The variation in permittivity affects only the detector quality factor, $Q_{\text {det }}$, of the LEKIDs as the filters are inductively coupled to the feedline and have a resulting immunity to the dielectric constant, whereas the coupling between the filter and mm-wave absorber is predominantly capacitive. The dielectric thickness on the other hand, effects both the feedline and filter impedance resulting in a modification of both $Q_{\text {feed }}$ and the coupling to the mm-wave absorber. Control of this dimension on the $<3 \%$ level is required to ensure that it is not the dominant cause of scatter in the resonator 


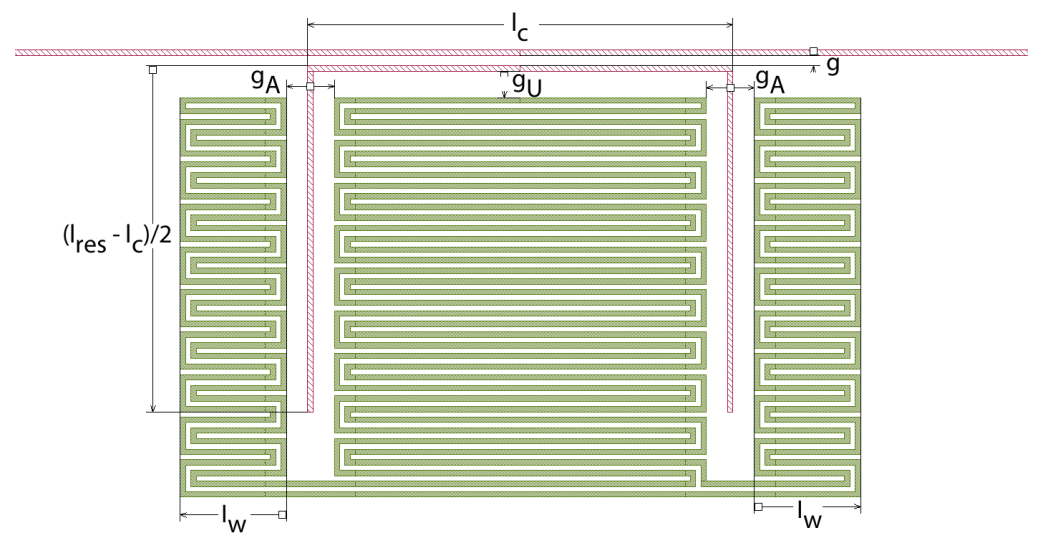

Figure 4. Parametric simulation layout for the tolerance analysis.

Table 1. Tolerances for an $\mathcal{R}=700$ microstrip resonator on $0.5 \mu \mathrm{m}$ silicon nitride

\begin{tabular}{ccc|cccc}
\hline \hline & & & \multicolumn{4}{|c}{ Fractional change } \\
Variable & Description & Change & Freq & $\mathbf{Q}_{\mathbf{i}}$ & $\mathbf{Q}_{\mathbf{c}}$ & $\mathbf{Q}_{\mathbf{r}}$ \\
\hline$l$ & resonator length & $0.1 \mu \mathrm{m}$ & $1 \times 10^{-3}$ & - & - & - \\
$g$ & feed to res gap & $0.1 \mu \mathrm{m}$ & $8 \times 10^{-5}$ & - & 0.19 & 0.09 \\
$g_{A}$ & absorber to res gap & $0.1 \mu \mathrm{m}$ & $4 \times 10^{-5}$ & 0.05 & - & 0.03 \\
$w$ & Nb line width & $0.1 \mu \mathrm{m}$ & $3 \times 10^{-3}$ & 0.06 & 0.11 & 0.08 \\
$w_{A}$ & TiN line width & $0.1 \mu \mathrm{m}$ & $6 \times 10^{-5}$ & 0.06 & - & 0.03 \\
$d x$ & Nb/TiN offset in $x$ & $0.1 \mu \mathrm{m}$ & $5 \times 10^{-6}$ & 0.006 & - & 0.003 \\
\hline$R_{\mathrm{TiN}}$ & Resistivity & $20 \%$ & $6 \times 10^{-5}$ & 0.03 & - & 0.02 \\
$\epsilon_{N}$ & SiN permittivity & $2.5 \%$ & $8 \times 10^{-3}$ & 0.05 & 0.004 & 0.02 \\
$T_{N}$ & SiN thickness & $2.5 \%$ & $5 \times 10^{-4}$ & 0.07 & 0.09 & 0.08 \\
$\tan \delta$ & SiN loss & $+10^{-4}$ & - & 0.04 & - & 0.02
\end{tabular}

$Q$.

Preliminary simulations suggest several approaches which can relax these tolerances even further, including designs in which multiple mm-wave resonators are coupled to a single LEKID absorber, and designs which make use of a second intermediate $n \lambda / 2$ resonator between the filter channel and LEKID. ${ }^{16}$

\section{LEKID DESIGN}

On resonance, the input impedance of the filter is zero (assuming that the internal resistance is zero) resulting in a purely real input impedance $Z_{\text {in }}=R_{d}$ (cf. Figure $5(\mathrm{~b})$ ), from the contribution of the LEKID. Consequently, a single isolated filter coupled to a well matched LEKID has a maximum absorption in a single pass of $50 \%$, as shown by the dashed red curve in Figure 6(b). Maximum absorption occurs when $R_{d}=Z_{0}$, or equivalently, $Q_{\text {det }}=Q_{\text {feed }}$, which from Equations 2 and 3, leads to

$$
Q_{\text {feed }}=2 \mathcal{R},
$$

which determines the required value of $Q_{\text {feed }}$ for the filters for a given spectral resolution.

Recently the exploration of superconducting nitride materials has revealed some of the exciting properties of titanium nitride (TiN) for use in micro-resonator devices. TiN LEKIDs have achieved internal quality factors as high as $3 \times 10^{7}$, enabling extremely high detector sensitivities. ${ }^{17}$ Moreover, the ability to tune the superconducting 


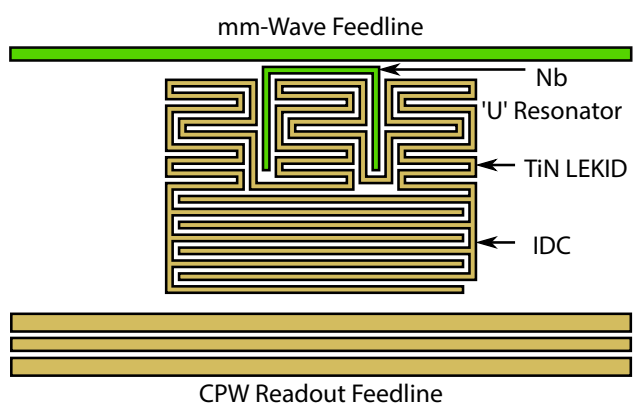

(a) Schematic layout of a single filter channel and TiN LEKID.

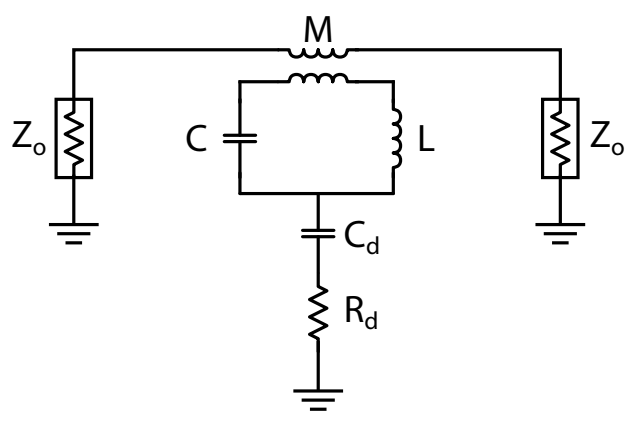

(b) Equivalent circuit diagram for a single filter channel. At mm-wave frequencies, the LEKID can be modelled as a lumped resistance.

Figure 5. Schematic of a single filter coupled to a LEKID.

critical temperature of TiN by varying the nitrogen content during the film deposition allows fine control over the material performance, making TiN an extremely attractive material for LEKID technology and a good candidate for use in the spectrometer.

To maximise the number of resonators in a given electronic bandwidth whilst accounting for a degree of non-ideal scatter in the resonant frequencies due to cross coupling, fabrication defects...etc., a baseline internal quality factor of $Q_{i}=10^{5}$ has been set for the SuperSpec TiN LEKIDs. ${ }^{18}$ To further increase the multiplexing density, the resonant frequency of the LEKIDs can be reduced down to the RF (100-500 MHz) range, which can be achieved by simply increasing the inductance and/or capacitance of the LEKID. This can be achieved by modifying the LEKID geometry, as well as taking advantage of the high kinetic inductance fraction of TiN. Furthermore, reducing the operating frequency of the LEKIDs has the added advantages of reducing the two-level system noise ${ }^{19}$ and reducing the cost and complexity of the readout electronics. ${ }^{11,12}$ Coupling from the filter to

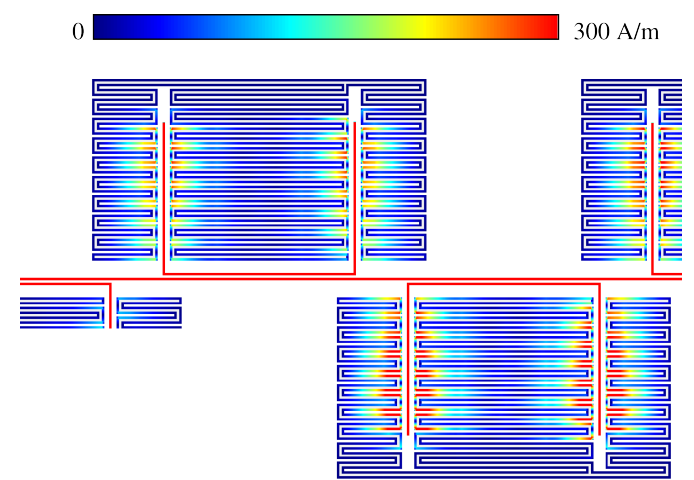

(a) Simulated current density at the resonant frequency of the centre filter.

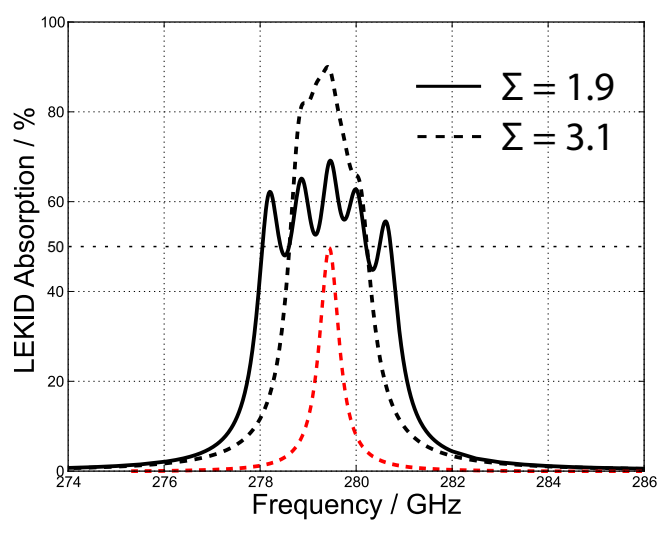

(b) Simulated absorption for two different values of $\Sigma$ demonstrating how the efficiency can be increased above $50 \%$ of a single isolated matched resonator (red dashed line).

Figure 6. Simulation results of a five-element filter bank.

the LEKID, described by $Q_{\text {det }}$, is determined by properties of the TiN and the geometry of the LEKID inductor. The TiN properties are constrained by the required sensitivity (e.g. $\left.T_{c}, V_{\text {ind }}\right){ }^{15}$ The inductor geometry (cf. 
Figure 5(a)) consists of a double wrapped meander, which has been shown to reduce the effect of microwave cross coupling between resonators, ${ }^{20}$ a crucial design consideration in order to maintain uniformity between pixels.

From the mm-wave point of view, the inductive meander can be decomposed into a set of resistive strips each capacitively coupled to the filter tines by a gap $g_{A}$ (cf. Figure 4 ). The length of the strips is determined by the decay length of TiN such that the opposite end of the strip can be viewed as a virtual ground as the current will have decayed to zero. The lumped resistance in Figure 5(b) is made up of the parallel combination of all the resistive strips and for a given value of $g_{A}$, the required number of strips to maximise the absorber coupling is set by the sheet resistance.

These relations, with minor adjustments to account for the coupling between neighbouring resistive strips, should allow for the design of a fully optimised mm-wave circuit. In practice, the SuperSpec prototype design has been driven by the desire to maximise the TiN volume into which mm-wave energy is deposited in order to produce a pixel with suitably low readout frequencies and high absorption efficiency. We therefore choose the densest TiN meander possible for all devices, and then interpolate from a series of Sonnet simulations to choose appropriate values of $f_{0}, Q_{\text {feed }}$, and $Q_{\text {det }}$ in order to achieve specific values of $g, l_{\text {res }}$, and $g_{A}$. Figure 6 shows the results of a five-element filter-bank now coupled to a matched absorber.

Figure 6(a) shows the current density in the mm-wave absorber at the resonant frequency of the bottom resonator. As discussed, power is capacitively coupled into the absorber and the current decays to zero on a characteristic length scale associated with the mm-wave loss in the TiN. Figure 6(b) shows the a plot of the inferred absorption in the LEKID for two different values of $\Sigma$. Absorption of more than $50 \%$ of incoming power across the band can be achieved by spacing the resonant frequencies of the filters closer together than the width of an individual channel. With a higher value of the oversampling factor, $\Sigma$, one can achieve arbitrarily efficient total absorption, at the cost of more readout channels. This effect can be seen in Figure 6(a), where incoming radiation at the centre frequency of the bottom resonator excites a (smaller) current in the neighbouring channel.

\section{HORN COUPLING DESIGN}

The mm-wave line is coupled to the telescope optics through a multiple-flare angle horn. ${ }^{21}$ This antenna design offers performance comparable with a corrugated horn while being simple to fabricate. The circular waveguide output for the antenna transitions into single mode oval waveguide to couple to a waveguide probe as shown in Figure 7(a). This probe is fabricated on the 25um thick device layer of the SOI wafer which supports the spectrometer chip. The radial stub probe transitions through a CPW transmission line to form the broadband impedance match between the waveguide and the microstrip of the spectrometer. By careful design of the ground plane of the waveguide probe, coupling to higher order modes in the probe channel was suppressed without the need of wire-bonds or beamleads. ${ }^{22}$ Simulation of this design show a coupling efficiency above $90 \%$ from 190 to $310 \mathrm{GHz}$ (cf. Figure 7(b)).

\section{PROTOTYPE TEST DEVICE}

The first fabricated prototype chip does not include the horn coupling structures and is suitable only for dark tests; however, it does include a full mm-wave filter-bank and feedline, and the same mask set can be readily modified to allow for optical tests. The mm-wave circuit includes $74 \mathrm{~mm}$-wave filters, 3 inline broad-band absorbers, and a terminating absorber. The 74 tuned filters span the $200-300 \mathrm{GHz}$ band and include both isolated individual filters and groups of five which are placed close in groups with a range of oversampling factors $(\Sigma)$. Expected values of $Q_{\text {det }}$ and $Q_{\text {feed }}$ independently sample the range from 600 to 2800, and include the design goal of $2 \mathcal{R}=1400$. The inline broad-band detectors consist of a short $\left(<\lambda_{\min } / 4\right)$ feedline meander which is proximate to a TiN absorber, similar to that used in the tuned filters. Sonnet simulations predict roughly $0.5 \%$ absorption across the full optical band with a slowly varying frequency dependence for these devices, which may be useful in characterising the response of the devices. The terminating absorber consists of several centimetres of meandered feedline surrounded by TiN with a $1 \mu \mathrm{m}$ separation, designed to absorb any radiation which arrives at the end of the feed and reduce reflections to $<20 \mathrm{~dB}$. Four long segments of the terminating absorber are used as the inductors for an additional set of broad-band absorber KIDs. Furthermore, these broad-band detectors 


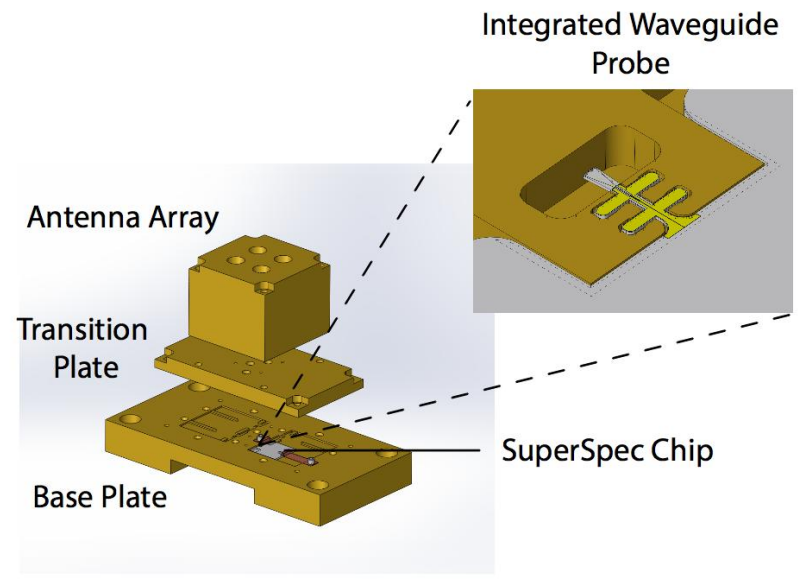

(a) CAD model of a $2 \times 2$ integrated horn array and packaging for SuperSpec.

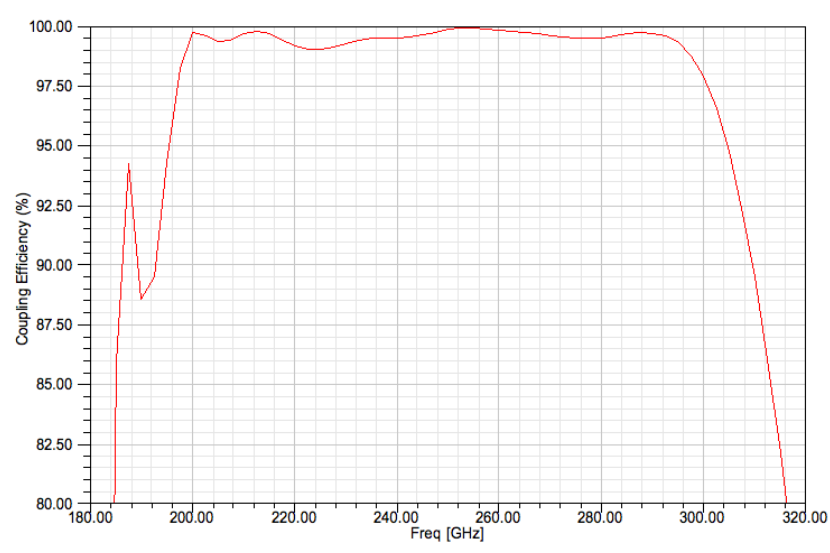

(b) Simulated performance of the waveguide-to-microstrip transition.

Figure 7. SuperSpec mounting assembly.

will provide a quantitive measure of the power that is not absorbed by the spectrometer; a potentially valuable diagnostic tool when characterising the performance of the filter-bank.

\section{CONCLUSION}

SuperSpec is a is novel, fully planar on-chip spectrometer based on a superconducting filter-bank made up of half wavelength filters. Titanium nitride lumped element kinetic inductance detectors are used to read out the power contained in each filter element, which allow a large amount of detectors to be read out on a single pair of coaxial transmission lines. Detailed electromagnetic simulations of the microstrip filter-bank demonstrate that a $\mathcal{R}=700$ spectrometer covering the $190-310 \mathrm{GHz}$ band is well within the capabilities of the JPL fabrication facility.

\section{ACKNOWLEDGMENTS}

This work has been supported in part by a NASA grant trough solicitation NNH10ZDA001N, task number 399131.02.06.03.43. P. S. Barry acknowledges the continuing support from the Science and Technology Facilities Council Ph.D studentship programme and grant programmes ST/G002711/1 and ST/J001449/1. E. Shirokoff, L. J. Swenson and C. M. McKenney acknowledge support from the W. M. Keck Institute for Space Studies. L. J. Swenson, M. I. Hollister and T. Reck acknowledge funding from NASA Postdoctoral Programme.

\section{REFERENCES}

[1] Eales, S., Dunne, L., Clements, D., Cooray, A., De Zotti, G., Dye, S., Ivison, R., Jarvis, M., Lagache, G., Maddox, S., Negrello, M., Serjeant, S., Thompson, M. A., Van Kampen, E., Amblard, A., Andreani, P., Baes, M., Beelen, A., Bendo, G. J., Benford, D., Bertoldi, F., Bock, J., Bonfield, D., Boselli, A., Bridge, C., Buat, V., Burgarella, D., Carlberg, R., Cava, A., Chanial, P., Charlot, S., Christopher, N., Coles, P., Cortese, L., Dariush, A., Da Cunha, E., Dalton, G., Danese, L., Dannerbauer, H., Driver, S., Dunlop, J., Fan, L., Farrah, D., Frayer, D., Frenk, C., Geach, J., Gardner, J., Gomez, H., Gonzalez-Nuevo, J., Gonzalez-Solares, E., Griffin, M., Hardcastle, M., Hatziminaoglou, E., Herranz, D., Hughes, D., Ibar, E., Jeong, W.-S., Lacey, C., Lapi, A., Lee, M., Leeuw, L., Liske, J., Lopez-Caniego, M., Muller, T., Nandra, K., Panuzzo, P., Papageorgiou, A., Patanchon, G., Peacock, J., Pearson, C., Phillipps, S., Pohlen, M., Popescu, C., Rawlings, S., Rigby, E., Rigopoulou, M., Rodighiero, G., Sansom, A., Schulz, B., Scott, D., Smith, D. J. B., Sibthorpe, B., Smail, I., Stevens, J., Sutherland, W., Takeuchi, T., Tedds, J., Temi, P., Tuffs, R., 
Trichas, M., Vaccari, M., Valtchanov, I., van der Werf, P., Verma, A., Vieria, J., Vlahakis, C., and White, G. J., "The Herschel ATLAS," arXiv.org astro-ph.CO (Oct. 2009).

[2] Oliver, S., Wang, L., Smith, A., Altieri, B., Amblard, A., Arumugam, V., Auld, R., Aussel, H., Babbedge, T., and Blain, A., "HerMES: SPIRE galaxy number counts at 250, 350 and 500 microns," arXiv.org (2010).

[3] Bradford, C., Ade, P., Aguirre, J., Bock, J. J., Dragovan, M., Duband, L., Earle, L., Glenn, J., Matsuhara, H., and Naylor, B., "Z-Spec: a broadband millimeter-wave grating spectrometer: design, construction, and first cryogenic measurements," Proceedings of SPIE 5498, 257-267 (2004).

[4] Hailey-Dunsheath, S., Nikola, T., Stacey, G. J., Oberst, T., Parshley, S., Benford, D. J., Moseley, S. H., and Staguhn, J. G., "ZEUS: A Submillimeter Grating Spectrometer for Exploring Distant Galaxies," in [American Astronomical Society Meeting Abstracts], Bulletin of the American Astronomical Society 36, 1401 (Dec. 2004).

[5] Ferkinhoff, C., Nikola, T., Parshley, S. C., Stacey, G. J., Irwin, K. D., Cho, H.-M., and Halpern, M., "ZEUS2: a second generation submillimeter grating spectrometer for exploring distant galaxies," arXiv.org astroph.IM (Oct. 2010).

[6] Day, P. K., LeDuc, H. G., Mazin, B. A., Vayonakis, A., and Zmuidzinas, J., "A broadband superconducting detector suitable for use in large arrays," Nature 425, 817-821 (2003).

[7] Doyle, S., Naylon, J., Mauskopf, P., Porch, A., and Dunscombe, C., "Lumped Element Kinetic Inductance Detectors," in [Proceedings of the Eighteenth International Symposium on Space Terahertz Technology], 170 (2007).

[8] Monfardini, A., Swenson, L. J., Bideaud, A., Yates, S. J. C., Benoit, A., Baryshev, A. M., Baselmans, J. J. A., Doyle, S., Klein, B., Roesch, M., Tucker, C., Ade, P., Calvo, M., Camus, P., Giordano, C., Guesten, R., Hoffmann, C., Leclercq, S., Mauskopf, P., and Schuster, K. F., "NIKA: A millimeter-wave kinetic inductance camera," arXiv.org astro-ph.IM (Apr. 2010).

[9] Hollister, M., Czakon, N., Day, P., Duan, R., Glenn, J., Golwala, S., Leduc, H., Maloney, P., Mazin, B., and Nguyen, H., "An Update on MUSIC: A Kinetic Inductance Detector Camera for Sub/Millimeter Astrophysics at the Caltech Submillimeter Observatory," Twenty-First International Symposium on Space Terahertz Technology 1, 263-269 (2010).

[10] O'Brien, K., Mazin, B. A., McHugh, S., Meeker, S., and Bumble, B., "ARCONS: a highly multiplexed superconducting UV to near-IR camera," arXiv.org astro-ph.IM (Jan. 2012).

[11] Swenson, L. J., "A pathfinder instrument for on-sky demonstration of low-cost 350 micron imaging arrays," Proceedings of SPIE (in prep) (2012).

[12] McKenney, C., "Design considerations for a background limited 350 micron pixel array using lumped element superconducting microresonators,", Proceedings of SPIE (in prep) (2012).

[13] Van der Pauw, L., "The radiation of electromagnetic power by microstrip configurations," Microwave Theory and Techniques, IEEE Transactions on 25(9), 719-725 (1977).

[14] Abbosh, A. M., "Analytical closed-form solutions for different configurations of parallel-coupled microstrip lines," IET Microwaves, Antennas \& Propagation 3(1), 137 (2009).

[15] Zmuidzinas, J., "MKIDs for Superspec: Design Considerations." Caltech Memo (July 2011).

[16] Kovács, A., "SuperSpec: circuit design," Proceedings of SPIE (in prep) (2012).

[17] Leduc, H. G., Bumble, B., Day, P. K., Eom, B. H., Gao, J., Golwala, S., Mazin, B. A., McHugh, S., Merrill, A., Moore, D. C., Noroozian, O., Turner, A. D., and Zmuidzinas, J., "Titanium nitride films for ultrasensitive microresonator detectors," Applied Physics Letters 97, 2509 (Sept. 2010).

[18] Shirokoff, E., "MKID development for SuperSpec: an on-chip, mm-wave, filter-bank spectrometer," Proceedings of SPIE (in prep) (2012).

[19] Zmuidzinas, J., "Superconducting Microresonators: Physics and Applications," Annual Review of Condensed Matter Physics (2012).

[20] Noroozian, O., Day, P., and Eom, B., "Crosstalk reduction for superconducting microwave resonator arrays," IEEE Transactions on Microwave Theory and Techniques 60(5), 1235-1243 (2012).

[21] Leech, J., Tan, B. K., Yassin, G., Kittara, P., Wangsuya, S., Treuttel, J., Henry, M., Oldfield, M. L., and Huggard, P. G., "Multiple flare-angle horn feeds for sub-mm astronomy and cosmic microwave background experiments," Astronomy and Astrophysics 532, A61 (July 2011). 
[22] Kooi, J., Chattopadhyay, G., Withington, S., Rice, F., Zmuidzinas, J., Walker, C., and Yassin, G., "A fullheight waveguide to thin-film microstrip transition with exceptional RF bandwidth and coupling efficiency," International Journal of Infrared and Millimeter Waves 24(3), 261-284 (2003). 